\title{
Directional ratio based on parabolic molecules and its application to the analysis of tubular structures
}

\author{
Demetrio Labate, Burcin Ozcan, Manos Papadakis \\ Department of Mathematics, University of Houston
}

\begin{abstract}
As advances in imaging technologies make more and more data available for biomedical applications, there is an increasing need to develop efficient quantitative algorithms for the analysis and processing of imaging data. In this paper, we introduce an innovative multiscale approach called Directional Ratio which is especially effective to distingush isotropic from anisotropic structures. This task is especially useful in the analysis of images of neurons, the main units of the nervous systems which consist of a main cell body called the soma and many elongated processes called neurites. We analyze the theoretical properties of our method on idealized models of neurons and develop a numerical implementation of this approach for analysis of fluorescent images of cultured neurons. We show that this algorithm is very effective for the detection of somas and the extraction of neurites in images of small circuits of neurons.
\end{abstract}

\section{INTRODUCTION}

One of the main problems in image analysis is the extraction of shape characteristics. This problem is especially challenging in biomedical imaging, where the objects of interest are frequently very complex, have a large variability in size and the images contrast is often highly irregular.

In this paper, we are concerned with images of neuronal networks acquired using laser scanning microscopy. As each neuron consists of a blob-like cell body, called the soma, and many elongated processes called the neurites (including one axon and multiple dendrites), a first essential imaging task is to distinguish two classes of shapes: a class of rather isotropic shapes associated with the somas and a class of highly anisotropic shapes associated with the neurites. Needless to say, any notion of isotropy vs. anisotropy depends on scale at which the structures of interest are being considered. For instance, a thick tube can be regarded within a small image patch as an isotropic structure, but, if is seen as a part of a bigger patch, its anisotropy becomes evident. As a last note, we remark that an additional feature plays a significant role in such images: the directionality of an object, which can be intuitively described as the direction of the "principal" axis, if any, of the restriction of an image within a certain image patch. Again, intuitively speaking, all these features taken together can use to characterize anisotropy.

In order to distinguish anisotropic objects, such as vessel-like structures, from isotropic ones, e.g disk-like shaped objects, we introduce the notion of Directional Ratio which is a precise measure of anisotropy of a structure within a certain scale. This new concept can be defined for every transform or analysis operator induced by families of directional atoms.

Although the following presentation is restricted to the 2-D setting, all concepts and development of the theory can be extended verbatim to 3-D. Furthermore, directional ratio can be computed for any image. However, our theoretical analysis of this new concept will be limited to a class of idealized images which we call cartoon neurons and are defined as functions of the form $\chi_{A}$, where $A$ is a subset of $\mathbb{R}^{2}$ (or $\mathbb{R}^{3}$ ) containing a union of rectangular and blob-like regions.

\section{A MULTISCALE MEASURE OF LOCAL ISOTROPY AND ANISOTROPY}

We start by introducing the following definition of local isotropy. Let $B(x, r)$ be a ball centered at $x$ of radius $r>0$.

Definition 2.1. Let $A \subset \mathbb{R}^{2}$. If $x \in A$ we say that the set $A$ is locally isotropic at location $x$ and at scale $s>0$ if $B(x, s / 2) \subseteq A$.

Obviously the scale at which the isotropy is observed is not unique. For example, let $A$ be a ball of radius $S>0$. Then the ball at its center is locally isotropic at any radius up to scale $2 S$. Our definition above attempts to establish a connection

E-mail: BO: bozcan@math.uh.edu, DL: dlabate@math.uh.edu, MP: mpapadak@math.uh.edu 


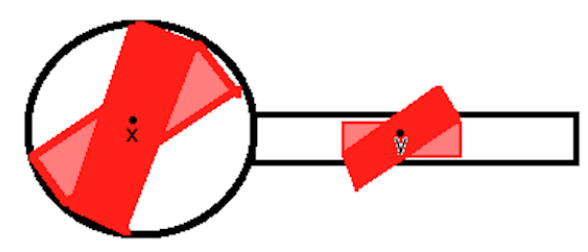

Figure 1. Cartoon neuron with a ball-shaped soma and a cylindrical neurite. The red rectangles are Haar-like directional molecules like the ones we use in our soma segmentation algorithm. Notice that regardless of the orientation the area of each rectangle centered at center of the soma contained in it is constant.

between the geometric property of the radius of a ball and a scale variable. Recall that the scale is a property of the physical domain defined in the Fourier domain in terms of dyadic decompositions introduced by Littlewood and Paley. Intuitively speaking, the spatial extent of an object dictates its "essential support" in the frequency domain which can be thought of as the subset of the frequency domain containing the essential support of the Fourier transform of the characteristic function of this isotropic patch. We will not attempt to discuss this connection in more detail here but we will return to it after Theorem 2.5.

To detect a region of local isotropy or, by contrast, a region of anisotropy we propose a novel concept requiring the use of molecules satisfying some appropriate directional sensitivity. For a function $f \in L^{2}\left(\mathbb{R}^{2}\right)$ we denote as $T_{y} f(x)=f(x-y)$, where $y \in \mathbb{R}^{2}$, the translation operator, as $\rho(R) f(x)=f(R x)$, where $R \in S O_{2}$ is an orthogonal matrix, the rotation operator and as $D_{M}^{j} f(x)=|\operatorname{det} M|^{j / 2} f\left(M^{j} x\right)$, where $M \in G L_{2}(\mathbb{R})$ is an invertible matrix, the dilation operator.

Definition 2.2. Suppose that $j>0$ and $x \in R^{2}$ is point in the support of the image $f \in L^{2}\left(\mathbb{R}^{2}\right)$. The Directional ratio $\mathscr{D}_{j} f(x)$ of the image $f$ at scale $j$ and point $x$ is given by

$$
\mathscr{D}_{j} f(x)=\frac{\inf _{R}\left\{\left|\left\langle f, T_{x} \rho(R) D_{M}^{j} \psi\right\rangle\right|: R \in S O(n)\right\}}{\sup _{R}\left\{\left|\left\langle f, T_{x} \rho(R) D_{M}^{j} \psi\right\rangle\right|: R \in S O(n)\right\}},
$$

where $M$ is anisotropic dilation matrix

$$
M=\left(\begin{array}{cc}
a & 0 \\
0 & a^{\frac{1}{2}}
\end{array}\right)
$$

and $a>1$ is a fixed constant (e.g., $a=2$ ).

We have the following useful result, which is illustrated in Figure 1.

THEOREM 2.3. Let A be a compact set with nonempty interior and $\psi$ be a measurable function such that $|\psi(x)| \leq$ $\frac{C}{(1+\|x\|)^{N}}$ where $N \geq 1+\delta, \delta>0$ and $\int_{\mathbb{R}^{n}} \psi(x) d x=1$. If $x_{0}$ is a point of local isotropy and $\varepsilon>0$, then there exists a scale $j>0$ such that $\mathscr{D}_{j} \chi_{S}\left(x_{0}\right)>1-\varepsilon$. [SOMETHING WRONG HERE. $\psi$ is not used and $\chi_{S}$ is not defined]

An example of a family of molecules [NOT CLEAR. No molecules used above] which satisfies the requirements of the previous theorem are the Directional Haar molecules defined, for $0<r<s$, by the characteristic functions

$$
\chi_{s, r}:=\chi_{\left[-\frac{s}{2}, \frac{s}{2}\right] \times\left[-\frac{r}{2}, \frac{r}{2}\right]^{n-1} .}
$$

We will use this family of molecules for our soma segmentation routines presented in Section 3.

As we show in Ref. 9, the converse of Theorem 2.3 is not true in general. This can also be illustrated computationally as indicated by the relatively high values of directional ratio at the boundary of the dendritic branch shown in the bottom right of each panel in Fig. 3.

A family $\left(m_{\lambda}\right)_{\lambda \in \Lambda}$ is called a family of parabolic molecules ${ }^{4}$ of order $\left(Q, M, N_{1}, N_{2}\right)$ if it can be written as

$$
m_{\lambda}(x)=2^{3 s_{\lambda} / 4} a^{(\lambda)}\left(D_{2^{s} \lambda} R_{\lambda}\left(x-x_{\lambda}\right)\right)
$$


such that

$$
\left|\partial^{\beta} \hat{a}^{(\lambda)}(\xi)\right| \leq C \min \left(1,2^{-s_{\lambda}}+\left|\xi_{1}\right|+2^{-s_{\lambda} / 2}\left|\xi_{2}\right|\right)^{M}\left(1+\|\xi\|^{2}\right)^{-N_{1} / 2}\left(1+\left|\xi_{2}\right|^{2}\right)^{-N_{2} / 2}
$$

for all $|\beta| \leq Q$. The constant $C$ is independent of $\lambda$.

The parabolic molecule-based Directional Ratio of $f \in L^{2}\left(\mathbb{R}^{2}\right)$ at scale $s_{\lambda}>0$ and $x \in \mathbb{R}^{2}$ is given by

$$
\mathscr{D}_{s_{\lambda}} f\left(x_{\lambda}\right)=\frac{\inf _{\lambda}\left\{\left|<f, m_{\lambda}>\right|\right\}}{\sup _{\lambda}\left\{\left|<f, m_{\lambda}>\right|\right\}} .
$$

It measures the strength of directional information at scale $s_{\lambda}$ and location $x$. The next theorem predicts consistent responses of directional ratio on tubular structures in general.

Curvelets $^{1}$ and shearlets ${ }^{2,5}$ are typical examples of parabolic molecules. However, in the shearlets construction orientation is determined by the powers of the shearing matrix, which plays a role very similar to rotation. Our first attempt to extend the concept of directional ratio to parabolic molecules focused on shearlets. For this purpose, in the following we use the shearlet transform $\mathscr{S} \mathscr{H}_{\psi}$. All notational conventions below can be found in ${ }^{6}$ where the theorem first appeared.

The shearlet-based Directional Ratio of $f$ at scale $j$ and location $x \in \mathbb{R}^{2}$ is given by

$$
\mathscr{D}_{j} f(x)=\frac{\inf _{s}\left\{\left|\mathscr{S}_{\mathscr{H}} f(j, s, x)\right|\right\}}{\sup _{s}\left\{\left|\mathscr{S}_{\mathscr{H}_{\psi}} f(j, s, x)\right|\right\}}, \quad f \in L^{2}\left(\mathbb{R}^{2}\right), x \in \mathbb{R}^{2},
$$

where $\mathscr{S}_{\mathscr{H}} f(a, s, x)=<f, T_{x} \psi_{j, s}>$ and the subscript $j, s$ are associated with the scale and shearing parameters, respectively. Disregarding notational details, one can immediately see that the shearlet-based directional ratio is a special case of the parabolic molecule-based directional ratio.

The next theorem predicts consistent responses of directional ratio on a 2-D vessel-like structure. Needless to mention that this concept and the following result can be extended to 3-D by using the 3-D shearlet representation.

THEOREM $2.4{ }^{6}$ Let $S_{0}$ be a cylinder with radius $r>0$ and infinite length in the direction of the positive y-half axis. Moreover, assume that the shearlet function $\psi$ is the tensor product of the 1-D molecules $\psi_{1}$ and $\psi_{2}$ satisfying the following properties:

1. The Fourier transforms of both $\psi_{1}$ and $\psi_{2}$ are $C^{\infty}$ and even;

2. The wavelet function $\psi_{1}$ has two "plateaus" in the frequency domain: $\hat{\psi}_{1}(\xi)=1$ for all $3 / 4 \leq|\xi| \leq 3 / 2$;

3. The "bump" function $\psi_{2}$ satisfies $\hat{\psi}_{2}(\xi)=c>0$ for all $|\xi| \leq 1 / 4$ and that $\int_{-L}^{L} \psi_{2}(x) d x>1$.

Then, for $4 r \leq 2^{-j} \leq 1 / 4$, there exists a threshold $\tau<1 / 3$ such that the shearlet-based Directional Ratio of $f$, given by (2), satisfies:

$$
\mathscr{D}_{j} f(y) \leq \tau \quad y=\left(y_{1}, y_{2}\right) \quad \text { sufficiently inside } S_{0}
$$

Let us now consider the more general case of parabolic molecules and the generalization of the previous theorem.

THEOREM 2.5. Let $S_{0}$ be a cylinder whose axis is parallel to the $y$-axis and has infinite length and radius $r$, where $2^{-7 s_{\lambda} / 4}<r<\frac{2^{-3 s_{\lambda} / 2}}{4}$ for some scale $s_{\lambda} \geq 9$.

Let, $a^{(\lambda)}$ be a parabolic molecule of order $\left(Q, M, N_{1}, N_{2}\right)$ satisfying the following conditions:

- $\int_{-2^{s} \lambda / 2}^{2^{s} \lambda / 2} \int_{-2^{s} \lambda / 2}^{2^{s} / 2} \widehat{a^{(\lambda)}}\left(\xi_{1}, \xi_{2}\right) d \xi_{1} d \xi_{2}>\frac{8}{10}\left\|\widehat{a^{(\lambda)}}\right\|_{1}$

- There exist constant $K_{1}>0$ and $\delta>0$, that does not depend on $\lambda$ such that for every scale $s_{\lambda} \geq 9$ we have $K_{1} 2^{s_{\lambda}(1+\delta)} \leq\left\|\widehat{a^{(\lambda)}}\right\|_{1}$ 

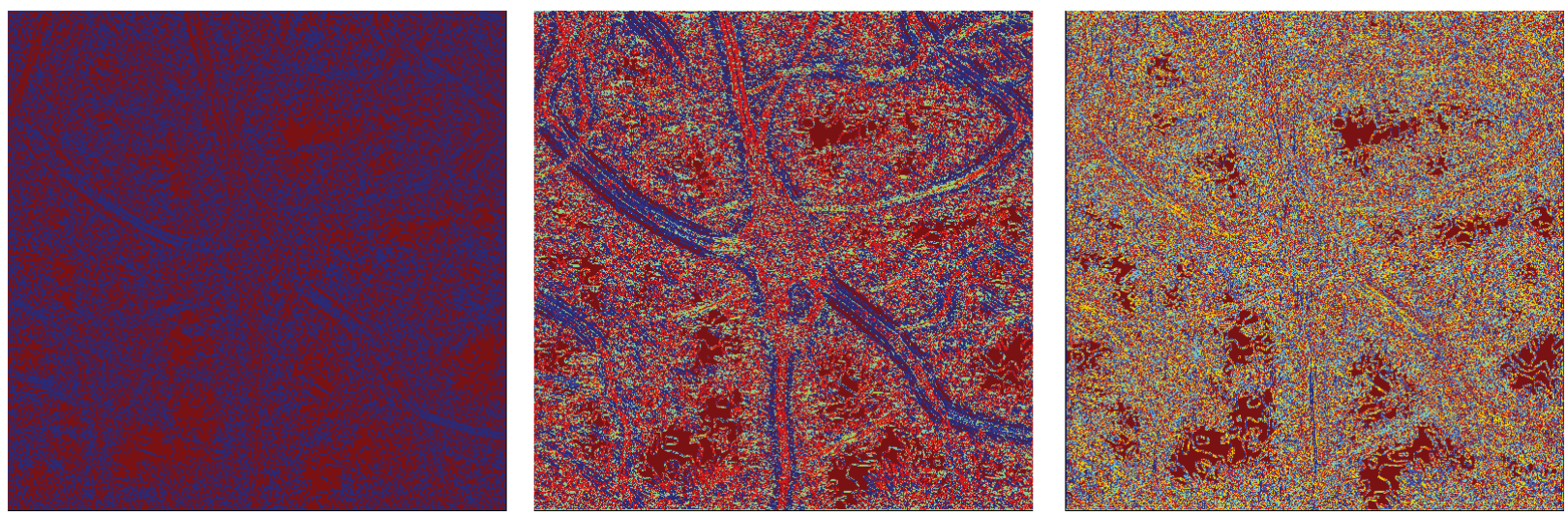

Figure 2. Values of directional ratio (color-coded) computed at various discrete scales; from left to right: $2^{-4} 2^{\frac{1}{4}}, 2^{-3} 2^{\frac{1}{4}}$, and $2^{-1} 2^{\frac{1}{4}}$ according to the conventions of. ${ }^{6}$ As the scale becomes coarser, the values of the directional ratio in the interior of neurites become consistently more uniform and do not exceed a certain low threshold, whose existence is predicted by Theorem 2.4. In the interior of the soma and of thicker neurites, the values of directional ratio vary wildly, once again as predicted by Theorem 2.4.

Then, $\mathscr{D}_{\lambda} \chi_{S_{0}}\left(x_{\lambda}\right) \leq 1 / 3$ for all grid points $x_{\lambda}$ in $S_{0}$.

Remark: The first of the two conditions of Theorem 2.5 is a weaker version of Conditions 2 and 3 of Theorem 2.4, while the Condition 2 shows that the $L^{1}$-norm of $\widehat{a^{(\lambda)}}$ is at the order of $2^{j(1+\delta)}$. Note that the existence of a positive constant $K_{2}$ such that $\left\|\widehat{a^{(\lambda)}}\right\|_{1} \leq K_{2} 2^{s_{\lambda}(1+\delta)}$ follows from Cauchy-Scwhartz inequality.

On isotropic regions, directional ratio values can be inconsistent because the values of the numerator and denominator of (1) may vary arbitrarily due to the approximate vanishing moment condition. This effect is due to the vanishing moment condition and can be seen in the middle and right panels of Fig. 2; due to the thickness of middle diagonal dendritic branch stretching from top left to bottom right of the panel, the values of directional ratio ranges randomly between zero and 1 . On the other hand, the values of directional ratio inside the same branch shown in the left panel of Fig. 2 are small because the scale and aspect ratio are consistent with the thickness of the branch. Stretching from top left to bottom right of the panel the values of directional ratio range randomly between zero and 1 . On the other hand, the values of directional ratio inside the same branch shown in the left panel are small because the scale and aspect ratio are consistent with the thickness of the branch. The aspect ratio is indirectly determined by the ratio of $L$ introduced in Condition 3 of Theorem 2.4 and $2 r$ the thickness of the neurite. Here the thickness directly determines the suitable scales. This is also true in Theorem 2.5. However, in this theorem, we have $2^{-7 s_{\lambda} / 4}<r<\frac{2^{-3 s_{\lambda} / 2}}{4}$. Combined with the fact that Conditions 2 and 3 of Theorem 2.4 are now replaced by the first condition of Theorem 2.5, this suggests that the aspect ratio of the tubular structure inside an image patch and its thickness in the same are both determined by $2^{-7 s_{\lambda} / 4}<r<\frac{2^{-3 s_{\lambda} / 2}}{4}$ and the first condition of Theorem 2.5. The combination of both plays a key role in the sensitivity of parabolic molecule-based directional ratio to the presence of tubular structures.

\section{SOMA SEGMENTATIONS IN 2-D}

In this section we illustrate an application where we use the directional ratio concept in order to segment blob-like regions automatically. The flowchart of our algorithm is shown in Fig. 3 and consists of the following steps: (1) a preprocessing routine to denoise the image; (2) a segmentation routine to separate the neurons from the background and to prepare the data for the next processing steps; (3) a routine that extracts the somas from the segmented images and includes Directional Ratio and level set routines; (4) a routine to separate those somas that are clustered together.

We here describe the 2D Soma Segmentation Algorithm which is the step where our directional ratio method plays a major role.

The input image of this step is the binary segmentation where the foreground structure (somas, dendritic branches and axons) was separated from the background. We consider here 2D images computed as maximum projections of the original 

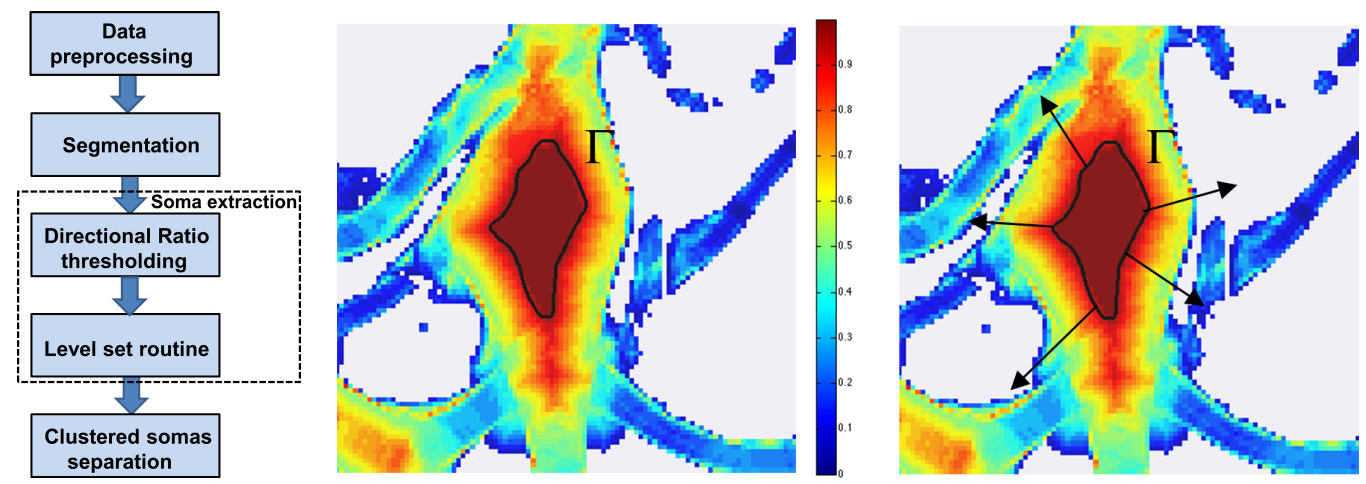

Figure 3. Left: Proposed algorithm for 2D soma detection. Right: Application of level set method to detect soma area. The figure shows a detail from a segmented image of a neuron (MIP) where colors correspond to the values of the Directional Ratio values and range between 1 (=red) and 0 (=blue). The application of the threshold value 0.9 identifies a region strictly inside the soma, with boundary curve $\Gamma$ (in the left panel). The level set method evolves the boundary curve $\Gamma$ with a velocity in the normal direction (indicated by the arrows in the right panel) depending on the magnitude of the gradient of Directional Ratio.
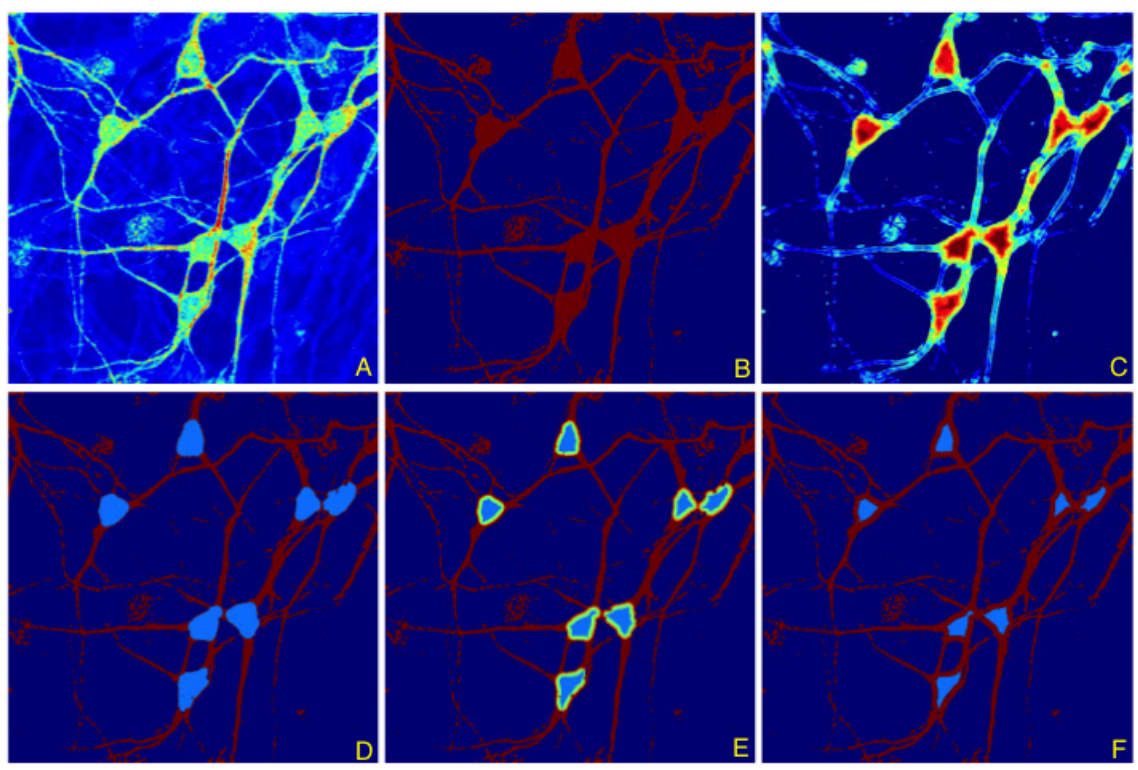

Figure 4. Illustration of the pipeline of the 2D soma detection algorithm (A) Denoised image, obtained using a shearlet-based denoising routine. ${ }^{3,8}$ (B) Segmented binary image. (C) Directional Ratio plot; the values range between 1, in red color (corresponding to more isotropic regions), and 0 , in blue color (corresponding to more anisotripc regions); note that the Directional Ratio is only computed inside the segmented region, i.e., inside the red region in Panel B.(D) Soma detection, obtained by applying the level set method with the initialization curve determined by the boundary of points of local isotropy in each soma shown in Panel F.(E) Application of the level set method; showing soma region growing. (F) Detection of initial soma region, obtained by applying the threshold 0.9 to the values of the Directional Ratio in Panel C. 

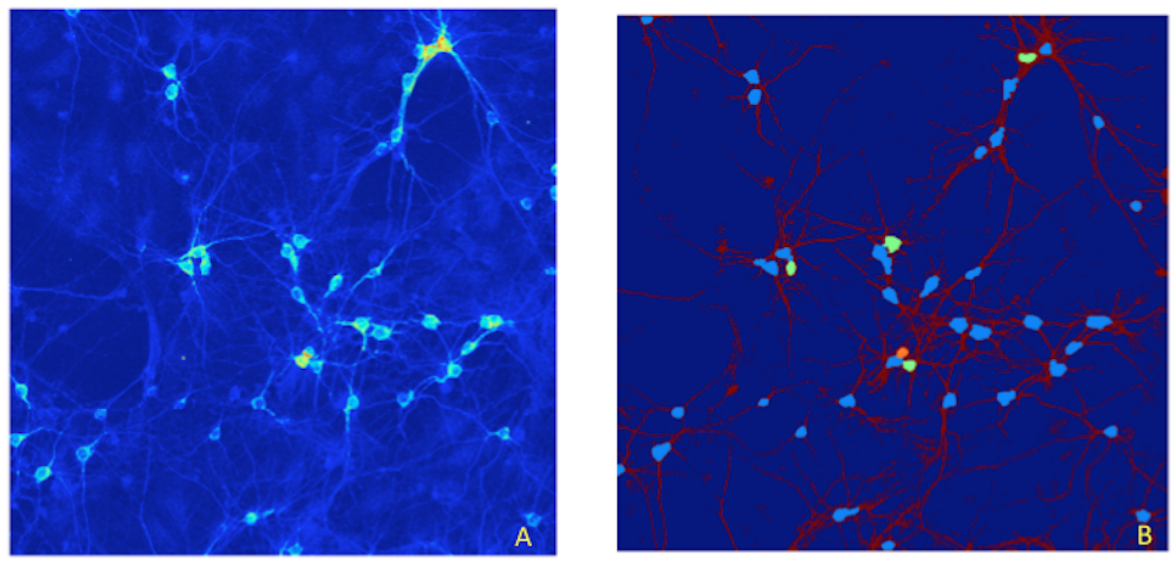

Figure 5. Example of 2D soma detection and separation of clustered somas on large field-of-view image. (A) Tiled and stitched confocal fluorescent image (MIP view) of a neuronal culture. Image size $=1894 \times 1894$ pixels $(1$ pixel $=0.28 \times 0.28 \mu \mathrm{m})$. $($ B $)$ Segmented binary image. Soma detection and separation of contiguous somas. Segmented neurites are shown in red color. Detected somas are shown in light blue; in case of contiguous somas, the separated somas appear in green and orange colors.

confocal image stacks (typically 10-20 slices). As shown in Flowchart 3, soma extraction is implemented in two steps: After computing the directional ratio for a certain scale and thresholding it (e.g. keep points whose value is greater than 0.9 ), we derive a region inside the soma whose boundary is used to initialize a level set routine. This area contains points of local isotropy at a certain scale. To find those regions we apply a threshold close to 1 as predicted by Theorem 2.3 , because these contain the points of local isotropy inside each soma at a given scale. Once somas are segmented the remaining part of the foreground corresponds to neurites (dendrites and axons). The identification inside the foreground of regions of local isotropy at a certain scale also facilitate soma detections.

To compute directional ratio we need to set the parameters used to generate the directional molecules $\chi_{s, r}$ in Algorithm 1. First, we choose the support size of directional atoms according to the given segmented image. The images we consider are cultured neurons acquired under similar conditions. Therefore, their soma sizes have dimensions of the same order of magnitude and we can use a single scale to generate the directional molecules. After some experimentation, we observed that choosing the correct length/scale $s / 2$ and aspect ratio of the directional molecules $\chi_{s, r}$ plays a crucial role in the accuracy of soma segmentation. If we choose $s / 2$ less than the approximate radius of soma regions, we notice that the regions connecting soma and dendrites have high directional ratio. This increases false positives. On the other hand, if we choose $s / 2$ bigger than approximate radius of the soma regions, we notice that the size of the regions which have high directional ratio shrink excessively in a way that prevents the level set routine to properly identify soma boundaries. Therefore, we manually choose the of directional molecules to have length equal to the approximate diameter of the somas in a segmented image. The radius of the somas in the images we work is approximately equal to 20 pixels. Hence, we set the support size of the directional atoms to be equal to 20 pixels. We also need to set the number of rotations of the directional molecules. The aspect ratio of the directional atoms is given by $\tan \left(\frac{\pi}{2 \times \text { number of rotations }}\right)$. Since we would like to have an elongated molecule, we set this ratio to be equal to 0.1 . Hence we set the rotation number to be equal to $\frac{\pi}{2 \arctan (0.1)}$ which is approximately equal to 15 . After that we proceed to the algorithm 2 to segment the somas.

To segment somas we produce the gradient of directional ratio and we apply a standard level set routine. ${ }^{11}$ The next proposition proves why such a gradient exists in a weak sense for shearlets. The initial condition for this level set problem is determined by the boundary of each region containing the points of local isotropy in each soma, that is, the points for which the directional ratio for a given scale take a value greater than 0.9 .

Proposition 3.1. Assume that, for every point $y$ in the open and convex set $\Omega \subset \mathbb{R}^{2}$, there exists $\alpha>0$ such that $\left|\left\langle f, \psi_{a, s, y}\right\rangle\right|>\alpha$ (stays consistently away from zero). Then the shearlet-based Directional Ratio $\mathscr{D}_{a} f$, at scale $s_{\lambda}=a$, restricted on $\Omega$, satisfies a Lipschitz condition and, thus, it is weakly differentiable on $\Omega$. 


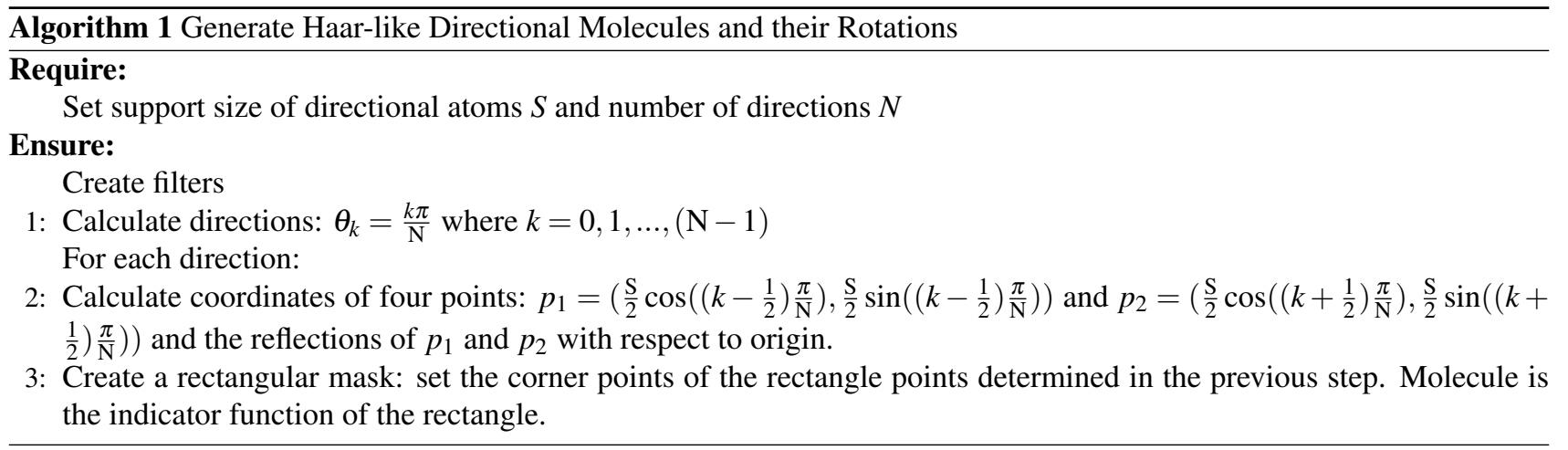

Note that this result can be generalized for any molecule, because the key idea of the proof is

$$
\begin{gathered}
\left|<f,\left(T_{h}-I\right) \psi_{a, s, y}>\right|=\left|\int_{\mathbb{R}^{2}} f(x)\left(\psi_{a, s, y}(x-h)-\psi_{a, s, y}(x)\right) d x\right| \\
\leq \int_{\mathbb{R}^{2}}\left|f(x)\left\|\psi_{a, s, y}(x-h)-\psi_{a, s, y}(x) \mid d x \leq C\right\| f\left\|_{1}\right\| h \|\right.
\end{gathered}
$$

which, for shearlets, follows from the Mean value theorem of differential calculus and only require that $\psi$ satisfies a Lipschitz condition a.e. Therefore, this condition applies to all parabolic molecules and to molecules satisfying the assumptions of Theorem 2.3. This justifies why we can compute the gradient field of directional ratio defined by Haar-like directional molecules in order to segment and detect somas.

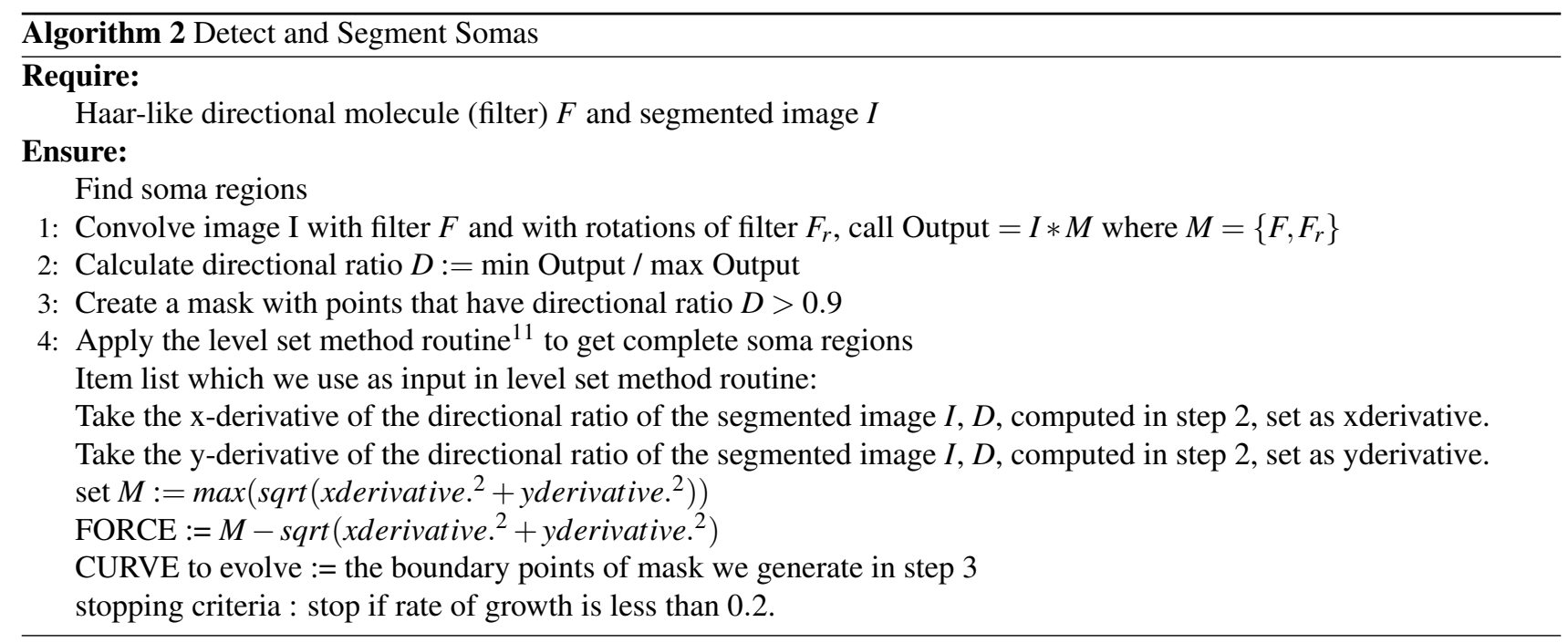

\section{3D SOMA DETECTION}

To detect soma surface and volume morphology in a confocal image stack of neuronal culture we use the soma region identified with Algorithm 2 in the $2 D$ segmented image.

Since image are obtained from cultured neurons, there are no overlapping somas along $z$ axis and this simplifies the localization of each soma in $3 D$. Hence, to localize somas in 3-D we construct a cylindrical mask whose 2-D boundary is coincides with the soma boundaries derived with Algorithm 2. To detect the soma surface of somas, we use a surface detection routine* , based on the shearlet transform, ${ }^{10}$ which we apply exclusively inside these cylindrical masks. Recall

\footnotetext{
* Source code of the surface detection routine is available at https://www.cs.umd.edu/users/oleary/software/
} 
that $3 D$ shearlets give rise to a representation system for $3 D$ data whose analyzing elements are surface-like waveforms with varying shape, 3 -D orientations and sizes, well-suited for accurate surface detection. ${ }^{10}$ Compared to other surface detection methods utilizing gradients, wavelets, Laplacians or Rayburst ${ }^{7}$ filters, shearlets provide smooth surface topology only at the cost of having to use a longer filter size to approximate the convolution action of 3D-shearlet molecules in the space domain.

One of the challenges in processing confocal image stacks is that bottom slices have low intensity values compared to surrounding background. Hence there is no well defined soma surface near bottom slices. Application of a conventional image enhancement approach is not very effective to improve the low signal-to-noise ratio of the images. This poses a difficulty for the 3-D shearlet-based surface detection algorithm.

To address this difficulty, we used a local thresholding routine to detect soma in these slices. This procedure is effective because it is applied in the already tightly localized soma region in 2-D. We next applied a morphological filling operator. Finally the detected surface from the top slices of the stack is morphologically filled and stitched with the bottom slices.

Some representative results of $3 D$ soma detection are shown in Figures 6.

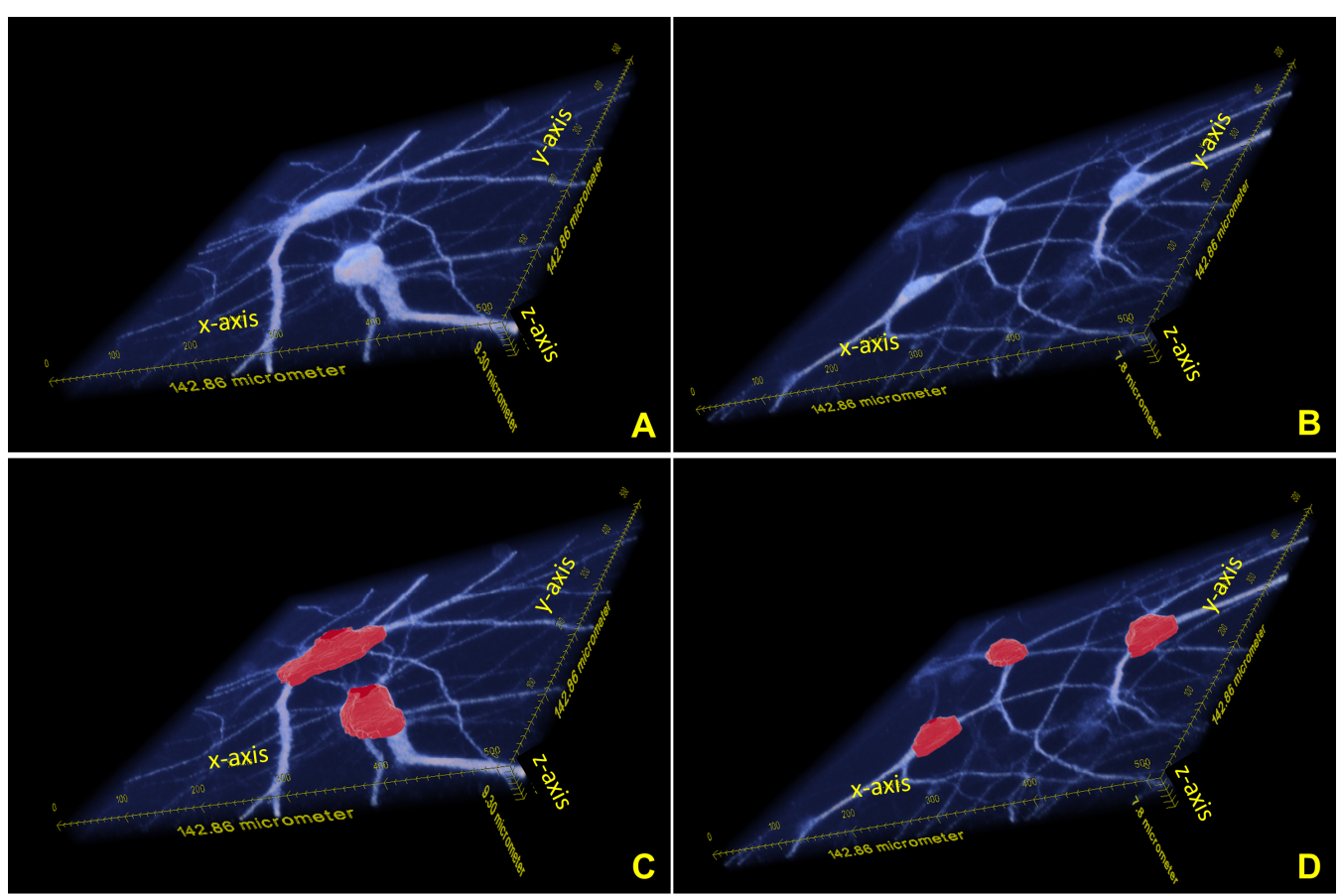

Figure 6. (A - B) Visualization of confocal image stacks of neuronal cultures. Note that stacks have a very limited extension along the $z$-direction where less that $10 \mu \mathrm{m}$ are available (25 - 30 optical slices). (C - D) The images illustrate, in red color, the detection of somas from the confocal image stacks.

\section{ACKNOWLEDGMENT}

This work was partially supported by NSD-DMS 1320910, 1008900, 1005799 and by a 2015 GEAR grant awarded by the University of Houston.

\section{REFERENCES}

1. E. J. Candès and D. L. Donoho. New tight frames of curvelets and optimal representations of objects with piecewise $C^{2}$ singularities. Commun. Pure Appl. Anal., 57(2):219-266, 2004. 
2. G. R. Easley and D. Labate. Image processing using shearlets. In Shearlets: Multiscale Analysis for Multivariate Data, Appl. Numer. Harmon. Anal., pages 283-325. Birkhäuser Boston, Boston, MA, 2012.

3. G. R. Easley, D. Labate, and WangQ Lim. Sparse directional image representations using the discrete shearlet transform. Appl. Comput. Harmon. Anal., 25:25-46, 2008.

4. Kutyniok G. Grohs P. Parabolic molecules. Found. Comput. Math., 14(2):299-337, 2014.

5. K. Guo and D. Labate. Optimally sparse multidimensional representation using shearlets. SIAM J. Math. Analysis, 39(1):298-318, 2007.

6. D. Labate, F. Laezza, P. Negi, B. Ozcan, and M. Papadakis. Efficient processing of fluorescence images using directional multiscale representations. Math. Model. Nat. Phenom., 4(1):1-3, January 2014.

7. S. Mallat. A Wavelet Tour of Signal Processing, Third Edition: The Sparse Way. Academic Press, 3rd edition, 2008.

8. P. S. Negi and D. Labate. 3-d discrete shearlet transform and video processing. IEEE Transactions on Image Processing, 21(6):2944-2954, 2012.

9. B. Ozcan, D. Labate, D. Jimenez, and M. Papadakis. Directional and non-directional representations for the characterization of neuronal morphology. In Wavelets XV (San Diego, CA, 2013), SPIE Proceedings, volume 8858, pages 1050-1053, 2013.

10. David A. Schug, Glenn R. Easley, and Dianne P. OLeary. Three-dimensional shearlet edge analysis. Proc. SPIE 8058, Independent Component Analyses, Wavelets, Neural Networks, Biosystems, and Nanoengineering IX, 2011.

11. B. Sumengen. Vision Research Lab at UC Santa Barbara. http://barissumengen.com/level_set_methods/index.html, UC Santa Barbara. 